\title{
A Second Proof of the Payne-Pólya-Weinberger Conjecture
}

\author{
Mark S. Ashbaugh ${ }^{1}$ and Rafael D. Benguria ${ }^{2 \star}$ \\ ${ }^{1}$ Department of Mathematics, University of Missouri, Columbia, MO 65211, USA \\ E-mail address: MATHMSA@UMCVMB.BITNET \\ ${ }_{2}$ Facultad de Física, P. Universidad Católica de Chile, Avda. Vicuña Mackenna 4860, \\ Casilla 306, Santiago 22, Chile \\ E-mail address: RBENGURI@PUCING.BITNET
}

Received September 3, 1991

\begin{abstract}
Without using product representations or elaborate comparisons of zeros we prove the two key properties of the Bessel function ratio $J_{p+1}\left(j_{p+1,1} x\right) / J_{p}\left(j_{p, 1} x\right)$ that we used to prove the Payne-Pólya-Weinberger conjecture. In these new proofs we use only differential equations and the Rayleigh-Ritz method for estimating lowest eigenvalues. The new proofs admit generalization to other related problems where our previous proofs fail.
\end{abstract}

\section{Introduction}

In our recent proof $[3,4]$ of the Payne-Pólya-Weinberger conjecture [12,13], the success of our method turned on certain technical results concerning Bessel functions. To be specific, two key properties of the function

$$
w(x)=\frac{J_{p+1}(\beta x)}{J_{p}(\alpha x)} \text { for } x \in[0,1]
$$

are used (at $x=0$ and $x=1$ we define $w(x)$ by its limiting values from within $(0,1)$ ), where $\alpha=j_{p, 1}$ and $\beta=j_{p+1,1}$ denote the first positive zeros of the Bessel functions $J_{p}(x)$ and $J_{p+1}(x)$ (our notation follows that of Abramowitz and Stegun [1]). The two properties are

(1) $w(x)$ is increasing for $x \in[0,1]$, and

(2) $B(x) \equiv w^{\prime}(x)^{2}+(2 p+1) w(x)^{2} / x^{2}$ is decreasing for $x \in[0,1]$.

* Partially supported by FONDECYT (Chile) project number 1238-90 
For the PPW conjecture in $n$ dimensions these properties are needed for the value $p=n / 2-1$; however, in this paper we shall treat all $p \geqq 0$ as this requires no additional effort. For more discussion concerning the PPW conjecture the reader should consult our papers [3,4]; this paper is directed only toward proving properties (1) and (2) above and then discussing various technical points concerning Bessel functions.

In our previous papers the proofs that we gave of (1) and (2) went by way of product representations for Bessel functions and a certain inequality between ratios of zeros of Bessel functions which we established using a somewhat technical differential comparison argument. This argument was quite involved and also proved substantially more than is actually needed since property (2) was obtained by showing that $w^{\prime}(x)$ and $w(x) / x$ are individually decreasing on $[0,1]$ (they are both nonnegative there). It therefore seemed to us that a simpler proof of (1) and (2) might be found. Actually property (1) is relatively easy to prove and even before the papers $[3,4]$ were written we had proofs of it which avoided the technical details alluded to above. Thus our main objective in this paper is to given an alternative proof of property (2). Along the way we also provide alternative proofs of (1).

Our new proofs are based only on a direct analysis of the behavior of $w(x)$ and $B(x)$ based on a simple (Riccati) differential equation satisfied by the function

$$
q(x)=\frac{x w^{\prime}(x)}{w(x)}
$$

and on inequalities between the first zeros of the Bessel functions $J_{p}(x)$ and $J_{p+1}(x)$ which we prove by simple Rayleigh-Ritz arguments (after all, the first zeros of Bessel functions give the first eigenvalues of certain naturally associated differential operators). Thus our new proofs entirely avoid the use of product representations and the inequalities comparing all the zeros of the Bessel functions $J_{p}(x)$ and $J_{p+1}(x)$ and work instead via the differential equations involved and inequalities between their first eigenvalues. This approach can be used to generalize our proof of the PPW conjecture in certain interesting directions which we discuss briefly in our concluding remarks (Sect. 5).

\section{The New Proofs}

With $q$ defined by (1.2) and $B$ as in property (2) above it is easy to see that

and

$$
B=\left[q^{2}+(2 p+1)\right](w / x)^{2}
$$

$$
B^{\prime}=2\left[q q^{\prime}+(q-1)\left(q^{2}+(2 p+1)\right) / x\right](w / x)^{2} .
$$

From (1.2) and (2.2) it is clear that properties (1) and (2) will follow if we can show that

and

$$
0 \leqq q \leqq 1
$$

$$
q^{\prime} \leqq 0
$$

hold for $0 \leqq x \leqq 1$. This is what we now proceed to do. For this we shall rely on a 
differential equation satisfied by $q$ for $0<x<1$, specifically,

$$
q^{\prime}=\left(\alpha^{2}-\beta^{2}\right) x+(1-q)(q+(2 p+1)) / x+2 \alpha q J_{p+1}(\alpha x) / J_{p}(\alpha x) .
$$

To arrive at this equation, first compute from (1.2) that

$$
q=x\left[\beta \frac{J_{p+1}^{\prime}(\beta x)}{J_{p+1}(\beta x)}-\alpha \frac{J_{p}^{\prime}(\alpha x)}{J_{p}(\alpha x)}\right]
$$

and then use the standard Bessel function identities

$$
J_{p+1}^{\prime}(x)=J_{p}(x)-\frac{p+1}{x} J_{p+1}(x)
$$

and

$$
J_{p}^{\prime}(x)=-J_{p+1}(x)+\frac{p}{x} J_{p}(x)
$$

to eliminate the derivatives of Bessel functions, obtaining

$$
q=\beta x \frac{J_{p}(\beta x)}{J_{p+1}(\beta x)}+\alpha x \frac{J_{p+1}(\alpha x)}{J_{p}(\alpha x)}-(2 p+1) .
$$

Next, differentiate (2.9), use (2.7) and (2.8) again to eliminate all derivatives of Bessel functions, and finally eliminate $J_{p}(\beta x) / J_{p+1}(\beta x)$ in favor of $q$ and $J_{p+1}(\alpha x) / J_{p}(\alpha x)$ using (2.9). One will then have (2.5).

Since we shall need the boundary behavior of $q$ at $x=0$ and $x=1$ (this is necessitated by the fact that the coefficients in the right-hand side of the differential Eq. (2.5) become singular at the two endpoints) we give these now. We find the following.

and

$$
q(0)=1, \quad q^{\prime}(0)=0, \quad q^{\prime \prime}(0)=\alpha^{2} /(p+1)-\beta^{2} /(p+2)
$$

$$
q(1)=0, \quad q^{\prime}(1)=\left(2 p+1+\alpha^{2}-\beta^{2}\right) / 3 .
$$

We next establish that $q^{\prime}(1)<0$ and $q^{\prime \prime}(0)<0$, which are necessary for (2.3) and (2.4) to hold.

Lemma 2.1. With notation as above, for $p \geqq 0$

$$
2 p+1+\alpha^{2}-\beta^{2}<0 \text {. }
$$

Hence $q^{\prime}(1)<0$.

Proof. We do this by a Rayleigh-Ritz variational argument. Since $\alpha^{2}$ is the first eigenvalue of the problem

$$
\frac{-1}{x}\left(x y^{\prime}\right)^{\prime}+\frac{p^{2}}{x^{2}} y=\lambda y \quad \text { on }[0,1]
$$

with the boundary conditions $y(0)$ finite and $y(1)=0$, we have

$$
\alpha^{2} \leqq \frac{\int_{0}^{1}\left[x\left(u^{\prime}\right)^{2}+p^{2} u^{2} / x\right] d x}{\int_{0}^{1} x u^{2} d x}
$$


for any nontrivial trial function $u$ which satisfies the boundary conditions and for which the integrals in (2.14) exist. Of course we know that $u_{1}=J_{p}(\alpha x)$ is the first eigenfunction of (2.13) and will give $\alpha^{2}$ exactly if substituted into (2.14). But instead of $J_{p}(\alpha x)$ we substitute $u=J_{p+1}(\beta x)$, which is a valid trial function. We obtain after an integration by parts and use of Bessel's equation for $J_{p+1}$ (Eq. (2.13) with $p \rightarrow p+1, \lambda \rightarrow \beta^{2}$ )

$$
\alpha^{2}<\beta^{2}-(2 p+1) \frac{\int_{0}^{1} \frac{1}{x} u^{2} d x}{\int_{0}^{1} x u^{2} d x}<\beta^{2}-(2 p+1),
$$

the last part because $1 / x>x$ on $[0,1)$.

Remark. If one uses a trial function $u=J_{r}\left(j_{r, 1} x\right)$ with $r>p$ here one obtains $j_{p, 1}^{2}<j_{r, 1}^{2}-\left(r^{2}-p^{2}\right)$, i.e. $j_{p, 1}^{2}-p^{2}$ is an increasing function for $p \geqq 0$. The well-known fact that $j_{p, 1}$ is an increasing function of $p$ also follows from this (so that, in particular, $\alpha<\beta$ ). We shall have occasion to use this several times in what follows, which we shall do with no particular comment.

Next we show $q^{\prime \prime}(0)<0$ which is somewhat more difficult to establish.

Lemma 2.2. With notation as above, for $p \geqq 0$,

Hence $q^{\prime \prime}(0)<0$.

$$
\frac{\alpha^{2}}{p+1}-\frac{\beta^{2}}{p+2}<0 \text {. }
$$

Proof. We use the Rayleigh-Ritz principle (2.14) again but this time with the trial function $u=J_{p+1}(\beta x) / x$. This does a better job of mimicking the behavior of the true wavefunction $u_{1}=J_{p}(\alpha x)$ at $x=0$, but correspondingly the argument becomes more delicate. Again it is convenient to work from the form

$$
\alpha^{2}<\frac{\int_{0}^{1} u\left[-\left(x u^{\prime}\right)^{\prime}+p^{2} u^{2} / x\right] d x}{\int_{0}^{1} x u^{2} d x} .
$$

Working as before, but this time using only (2.8) to eliminate the derivatives $J_{p}^{\prime}(x)$ and $J_{p+1}^{\prime}(x)$, one obtains

$$
\alpha^{2}<\beta^{2} \frac{\int_{0}^{1} u\left[-J_{p+3}(t)+2(p+1) J_{p+2}(t) / t\right] d x}{\int_{0}^{1} x u^{2} d x},
$$

where we have found it convenient to introduce the variable $t=\beta x$ in the upper integral. Establishing the lemma is now seen to be equivalent to showing that

$$
\frac{p+1}{p+2} \geqq \frac{\int_{0}^{1} u\left[-J_{p+3}(t)+2(p+1) J_{p+2}(t) / t\right] d x}{\int_{0}^{1} u J_{p+1}(t) d x}
$$


which is the same as

$$
\begin{aligned}
0 & \geqq \int_{0}^{1} u\left[-(p+1) J_{p+1}(t)-(p+2) J_{p+3}(t)+2(p+1)(p+2) J_{p+2}(t) / t\right] d x \\
& =-\int_{0}^{1} u J_{p+3}(t) d x=-\int_{0}^{1} \frac{1}{x} J_{p+1}(\beta x) J_{p+3}(\beta x) d x,
\end{aligned}
$$

and this is obviously true since $J_{p+1}(x)$ and $J_{p+3}(x)$ are both positive on $(0, \beta)$. The simplification between the second and third expressions in (2.19) is by use of the Bessel function identity $J_{p-1}(x)+J_{p+1}(x)=2 p J_{p}(x) / x$. Having established (2.19) the lemma is proved.

Remark. It is perhaps worth mentioning that the restriction to $p \geqq 0$ in Lemmas 2.1 and 2.2 can be avoided by considering in place of $(2.14)$ the variational principle

for the equation

$$
\lambda \leqq \frac{\int_{0}^{1} x^{2 p+1}\left(u^{\prime}\right)^{2} d x}{\int_{0}^{1} x^{2 p+1} u^{2} d x}
$$

$$
-\left(x^{2 p+1} u^{\prime}\right)^{\prime}=\lambda x^{2 p+1} u .
$$

The boundary conditions for admissible functions in this variational principle are $u$ finite at $x=0$ and $u(1)=0$. This variational principle is good for all $p>-1$ and gives back $\lambda_{1}=j_{p, 1}^{2}$ in the sense that $j_{p, 1}^{2}$ is the minimum of the right-hand side over all admissible functions. Equation (2.21) is related to Bessel's equation (2.13) via the change of variable $y=x^{p} u$. Hence, our choices of trial functions for (2.20) should be taken as $x^{-p}$ times our old trial functions. With this change effected, calculations can be carried out paralleling all those above but covering the larger class $p>-1$.

With the boundary behaviors out of the way, we turn back to considering the differential equation (2.5). To properly analyze this equation we need to have certain concavity/convexity results concerning the coefficient function $J_{p+1}(\alpha x) / J_{p}(\alpha x)$ at hand. These results are in the following two lemmas.

Lemma 2.3. $J_{p+1}(x) / J_{p}(x)$ is strictly convex on $[0, \alpha)$ for $p \geqq-1 / 2$.

Proof. Letting $y=J_{p+1}(x) / J_{p}(x)$ and using (2.7) and (2.8) again one readily finds

$$
J_{p}(x)^{2} y^{\prime}=J_{p}(x)^{2}+J_{p+1}(x)^{2}-(2 p+1) J_{p}(x) J_{p+1}(x) / x \equiv \theta(x),
$$

and in a similar fashion,

$$
[x \theta(x)]^{\prime}=(2 p+1) J_{p}(x) J_{p+1}(x) / x .
$$

Since $x \theta(x)=0$ at $x=0$ if $p>-1 / 2$ and $=2 / \pi$ if $p=-1 / 2$ and $J_{p}(x) J_{p+1}(x)>0$ in $(0, \alpha)$ it follows that $\theta(x)>0$ in $(0, \alpha]$. Moreover, from $(2.22)$ we find

Then from

$$
J_{p}(x)^{2} y^{\prime \prime}=\theta^{\prime}(x)+2 \frac{J_{p+1}(x)}{J_{p}(x)} \theta(x)-\frac{2 p}{x} \theta(x) \equiv s(x) .
$$

$$
s(x)=\frac{(2 p+1)}{x^{2}} J_{p}(x) J_{p+1}(x)+2 \frac{J_{p+1}(x)}{J_{p}(x)} \theta(x)-\frac{(2 p+1)}{x} \theta(x),
$$


yet another calculation, using (2.7), (2.8), and (2.23), yields

$$
\left[x^{3} s(x)\right]^{\prime}=2 x^{2} \theta(x)\left[2 \frac{J_{p+1}(x)}{J_{p}(x)}+x \frac{\theta(x)}{J_{p}(x)^{2}}\right]>0 \text { in }(0, \alpha) .
$$

Since $x^{3} s(x)=0$ at $x=0$, this implies that $s(x)>0$ in $(0, \alpha)$ and therefore, by (2.24), $y^{\prime \prime}>0$ there and the proof is complete.

Lemma 2.4. $J_{p+1}(x) / x J_{p}(x)$ is strictly increasing on $[0, \alpha)$ for $p \geqq-1 / 2$.

Proof. This follows easily from Lemma 2.3. Letting $r(x)=J_{p+1}(x) / x J_{p}(x)$ we have $r(x)=y(x) / x$ and hence

$$
r^{\prime}(x)=\frac{x y^{\prime}-y}{x^{2}} \equiv \frac{h(x)}{x^{2}} .
$$

Now $h^{\prime}=x y^{\prime \prime}>0$ on $(0, \alpha)$ by Lemma 2.3 , and since $h(0)=0$ it is clear from this that $h>0$ on $(0, \alpha)$, hence $r^{\prime}(x)>0$ there and we are done.

We can now prove our main result which completes our new derivation of properties (1) and (2) from the Introduction.

Theorem 2.5. With notation and hypotheses as above, for $x \in[0,1] 0 \leqq q \leqq 1$ and $q^{\prime} \leqq 0$, and hence $w$ is an increasing function there while $B$ is decreasing.

Proof. We begin by proving that $q \leqq 1$. Suppose not. Then we can find points $x_{1}, x_{2}$ with $0<x_{1}<x_{2}<1$ satisfying $q\left(x_{1}\right)=1=q\left(x_{2}\right)$ and $q^{\prime}\left(x_{1}\right) \geqq 0, q^{\prime}\left(x_{2}\right) \leqq 0$ and then using Eq. (2.5) we would have

$$
0 \leqq \frac{1}{x_{1}} q^{\prime}\left(x_{1}\right)=\alpha^{2}-\beta^{2}+\frac{2 \alpha}{x_{1}} \frac{J_{p+1}\left(\alpha x_{1}\right)}{J_{p}\left(\alpha x_{1}\right)}<\alpha^{2}-\beta^{2}+\frac{2 \alpha}{x_{2}} \frac{J_{p+1}\left(\alpha x_{2}\right)}{J_{p}\left(\alpha x_{2}\right)}=\frac{1}{x_{2}} q^{\prime}\left(x_{2}\right) \leqq 0
$$

a contradiction. The intermediate inequality here comes from Lemma 2.4. A similar consideration for $q=0$ using the fact that $(2 p+1) / x^{2}$ is decreasing shows that $q \geqq 0$ must also hold. Finally, to prove that $q^{\prime} \leqq 0$ on $(0,1]$ note that if not we could find three points $x_{1}, x_{2}, x_{3}$ such that $0<x_{1}<x_{2}<x_{3}<1$ and satisfying $q\left(x_{1}\right)=q\left(x_{2}\right)=$ $q\left(x_{3}\right), q^{\prime}\left(x_{1}\right)<0, q^{\prime}\left(x_{2}\right)>0$, and $q^{\prime}\left(x_{3}\right)<0$. Writing $x_{2}=\lambda x_{1}+(1-\lambda) x_{3}$, where $\lambda \in(0,1)$ and using the convexity of $1 / x,\left(\alpha^{2}-\beta^{2}\right) x$, and $J_{p+1}(x) / J_{p}(x)$ (from Lemma 2.3) we then have ( $q$ here will denote the common value of $q$ at the $3 x_{i}$ 's)

$$
0<q^{\prime}\left(x_{2}\right)=F\left(x_{2}, q\right)<\lambda F\left(x_{1}, q\right)+(1-\lambda) F\left(x_{3}, q\right)=\lambda q^{\prime}\left(x_{1}\right)+(1-\lambda) q^{\prime}\left(x_{3}\right)<0,
$$

a contradiction. Here we have used the strict convexity of $F(x, q)$ in $x$ for fixed $q$ where $F(x, q)$ denotes the right-hand side of the differential equation (2.5), i.e. $F(x, q)=\left(\alpha^{2}-\beta^{2}\right) x+(1-q)[q+(2 p+1)] / x+2 \alpha q J_{p+1}(\alpha x) / J_{p}(\alpha x)$. The conclusions concerning $w$ and $B$ now follow from Eqs. (1.2) and (2.2) since $w$ is known to be positive on $(0,1]$.

Remark. Our use of convexity in this argument is essentially the same as in our paper [2]. These arguments were inspired by more general arguments of this type that were used by Korevaar $[8,9]$ for partial differential equations. 
It is well to reflect on what we have gained with this new approach to properties (1) and (2). We have supplied proofs that do not use product representations and elaborate properties of zeros of Bessel functions. It turns out that it is possible to extend these new proofs to prove similar properties of other special functions such as Legendre or hypergeometric that arise when considering similar problems to the PPW conjecture in spaces of constant curvature. In these other situations, a product representation approach is not available. One has to make do, as was done above, with the differential equation, Rayleigh-Ritz eigenvalue estimates, and some special properties such as raising and lowering operations (like (2.7) and (2.8) for Bessel functions).

Finally, we note that this new approach still proves more than the bare essentials. $q>0$ on $(0,1)$ tells us that $w^{\prime}>0$ there since $w>0$. Also since $q=x w^{\prime} / w<1$ we have

$$
(w / x)^{\prime}=\frac{x w^{\prime}-w}{x^{2}}=w(q-1) / x^{2}<0,
$$

and hence also

$$
w^{\prime \prime}=[q(w / x)]^{\prime}=q^{\prime}(w / x)+q(w / x)^{\prime}<0,
$$

where use has been made of $q^{\prime} \leqq 0$ as well as (2.30). That is, we have again proved that $w / x$ and $w^{\prime}$ are individually decreasing. In our other proof attention was focused on the function $A(x)=w^{\prime} / w=q / x$ and we proved that $0<A<1 / x$ and $A^{\prime}<-1 / x^{2}$. Of these inequalities only $A^{\prime}<-1 / x^{2}$ does not seem to come out of our new proof. So in that sense our new approach is slightly weaker than our old. It might be noted in this regard that $A^{\prime}<-1 / x^{2}$ and $A(1)=0$ imply $A(x)>1 / x-1$ or, in terms of $q$, $q>1-x$, and, in particular, this implies $q^{\prime}(1) \leqq-1$, a strengthening of Lemma 2.1 above (see our last paragraph in Sect. 4 below for an even stronger result due to L. Lorch). However, one might still seek a proof along similar lines to the one given here that did not also prove the individual inequalities (2.30) and (2.31).

\section{Other Proofs that $w$ is Increasing}

As mentioned previously the fact that $w^{\prime}>0$ can be proved in a variety of relatively simple ways. These were not presented in $[3,4]$ because we were already using the product representation approach for proving property (2) and therefore we chose to give a uniform presentation of both properties from that viewpoint. Here we give two alternative proofs.

For the function $A(x)=w^{\prime}(x) / w(x)$ (mentioned at the end of the last section) we can compute

$$
A^{\prime}(x)=-A(x)^{2}-\left[\frac{1}{x}+2 \alpha \frac{J_{p}^{\prime}(\alpha x)}{J_{p}(\alpha x)}\right] A(x)-\left(\beta^{2}-\alpha^{2}\right)+\frac{2 p+1}{x^{2}}
$$

(another Riccati equation). From this it follows that whenever $A=0$

$$
A^{\prime}=-\left(\beta^{2}-\alpha^{2}\right)+\frac{2 p+1}{x^{2}}
$$


Also it is easy to find that $A(x)$ behaves like $1 / x$ for $x$ near zero and that $A(1)=0$ and $A^{\prime}(1)<0$. From these and the fact that $(2 p+1) / x^{2}-\left(\beta^{2}-\alpha^{2}\right)$ is decreasing it is clear that $A$ cannot reach 0 before $x=1$. Thus $A(x)>0$ for $x \in(0,1)$ and $w^{\prime}(x)=A(x) w(x)>0$ there follows. This argument could also be given in terms of $q$, working from Eq. (2.5), and indeed this is the argument given above.

Our second proof is also somewhat reminiscent of earlier proofs. We have

$$
A(x)=\beta \frac{J_{p+1}^{\prime}(\beta x)}{J_{p+1}(\beta x)}-\alpha \frac{J_{p}^{\prime}(\alpha x)}{J_{p}(\alpha x)}=\frac{\beta J_{p}(\alpha x) J_{p+1}^{\prime}(\beta x)-\alpha J_{p}^{\prime}(\alpha x) J_{p+1}(\beta x)}{J_{p}(\alpha x) J_{p+1}(\beta x)} .
$$

Calling the Wronskian in the numerator $N(x)$ one can compute that

$$
[x N(x)]^{\prime}=\left[\frac{2 p+1}{x^{2}}-\left(\beta^{2}-\alpha^{2}\right)\right] x J_{p}(\alpha x) J_{p+1}(\beta x) .
$$

Since $x J_{p}(\alpha x) J_{p+1}(\beta x)>0$ for $x \in(0,1)$ and the expression in square brackets is decreasing there this shows that either $x N(x)$ is increasing across the entire interval or is first increasing and then decreasing. (Which of the two possibilities occur depends of course on where $(2 p+1) / x^{2}-\left(\beta^{2}-\alpha^{2}\right)$ changes sign. We will see in what follows that this happens within $(0,1)$.) Now it is easily computed that $x N(x)$ takes the value 0 at $x=0$ if $p>-1 / 2$ (or $2 \sqrt{2} / \pi$ if $p=-1 / 2$ ) and also that $N(1)=0$ so that it must happen that $x N(x)$ first increases and then decreases and that $x N(x)>0$ for $x \in(0,1)$. This leads immediately to $A(x)>0$ and $w^{\prime}(x)>0$ for $x \in(0,1)$ as before.

\section{Additional Facts from the Product Representation Perspective}

In this section we cannot resist coming back to proofs using product representations even though we have avoided them thus far. In many problems involving Bessel functions, product representations together with properties of their zeros provide the most convenient and compact approach. It is when one wants to extend results obtained in this way to other related functions that this approach must usually be abandoned.

For example, our Lemmas 2.3 and 2.4 above are almost trivial from the product representation viewpoint. For Lemma 2.3 it is enough to compute

$$
\left[J_{p+1}(x) / J_{p}(x)\right]^{\prime \prime}=4 x \sum_{k=1}^{\infty} \frac{3 j_{p, k}^{2}+x^{2}}{\left(j_{p, k}^{2}-x^{2}\right)^{3}}>0 \text { for } x \in\left(0, j_{p, 1}\right) .
$$

Here $j_{p, k}$ denotes the $k^{\text {th }}$ positive zero of $J_{p}(x)$. This computation is begun by noting that $J_{p+1}(x) / J_{p}(x)=p / x-\left[\ln \left|J_{p}(x)\right|\right]^{\prime}$. Similarly, Lemma 2.4 is a direct consequence of

$$
\left[J_{p+1}(x) / x J_{p}(x)\right]^{\prime}=4 x \sum_{k=1}^{\infty} \frac{1}{\left(j_{p, k}^{2}-x^{2}\right)^{2}}>0 \text { for } x \in\left(0, j_{p, 1}\right) .
$$

The inequalities for $A(x)$ that we derived in [3,4] were substantially more difficult since the series obtained contained terms involving $j_{p, k}$ 's and also $j_{p+1, k}$ 's. The inequalities that allowed our proofs to work were comparison results for ratios 
of these zeros. Specifically these are

$$
\frac{j_{p+1, k}}{j_{p+1,1}}<\frac{j_{p, k}}{j_{p, 1}} \text { for } k=2,3,4, \ldots
$$

Since writing $[3,4]$ it has been brought to our attention by Martin E. Muldoon that such inequalities had been known for some time. In particular, the fact that $j_{p, k} / j_{p, 1}$ (and even $j_{p, k} / j_{p, l}$ for $k>l$ ) is decreasing with $p$ for $p>-1$ had been shown by L. Lorch $[10,11]$. His simple proof $[11]$ of this is to compute $\left[\ln \left(j_{p, k} / j_{p, 1}\right)\right]^{\prime}$ using the integral representation $[14$, p. 508 , Eq. (3)]

$$
\frac{d j_{p, k}}{d p}=2 j_{p, k} \int_{0}^{\infty} K_{0}\left(2 j_{p, k} \sinh t\right) e^{-2 p t} d t,
$$

where $K_{0}$ represents the standard modified Bessel function of order 0 (which is known to be decreasing on $[0, \infty)$ ). Yet another route to $(4.3)$ is provided by recent results of Gori, Laforgia, and Muldoon [6], who prove that $j_{v, k} / j_{\mu, k}$ is decreasing in $k$ for $v>\mu \geqq 1 / 2$. Related material on Bessel function zeros is to be found in [5] (which extends the results in [11]) and [7], to mention only two further articles from a large literature.

Finally, we mention that our result in Lemma 2.2 has also already been proved in a more general form. In [7], it is shown that $j_{p, 1}^{2} /(p+1)$ is an increasing function of $p$ for $p>-1$ (Theorem 2, p. 197). And with regard to Lemma 2.1 (see also the remark following it) we note that the stronger result that $j_{p, 1}^{2}-(p+1)(p+5)$ is positive and increasing for $p>-1$ has recently been proved by Lorch (private communication, 1991). In particular, this implies that $j_{p, 1}^{2}+2 p+7<j_{p+1,1}^{2}$ for all $p>-1$. We presented our proofs of Lemmas 2.1 and 2.2 above because they are simple, they give exactly the results needed for our proof of the PPW conjecture, and they point the way to extending our approach here to other related problems.

\section{Concluding Remarks}

The methods developed here permit generalization of our proof of the PaynePólya-Weinberger conjecture to the case of $\lambda_{2} / \lambda_{1}$ for the (Dirichlet) eigenvalues of the Laplacian on a domain contained in a hemisphere of $S^{n}$. In addition, one can use these same methods to obtain an isoperimetric result for the first nonzero Neumann eigenvalue of the Laplacian on a domain contained in a hemisphere of $S^{n}$. These results will be presented in subsequent papers on these topics, entitled "Sharp Upper Bound to the First Nonzero Neumann Eigenvalue for Bounded Domains in Spaces of Constant Curvature" and "Sharp Bound for the Ratio of the First Two Dirichlet Eigenvalues of a Domain in a Hemisphere of $S^{n}$."

Acknowledgements. We would like to thank Martin E. Muldoon and Lee Lorch for useful correspondence and valuable references to the literature concerning properties of Bessel function zeros. 


\section{References}

1. Abramowitz, M., Stegun, I. A.: Handbook of Mathematical Functions, National Bureau of Standards Applied Mathematics Series, vol. 55. U.S. Government Printing Office, Washington, D.C., 1964

2. Ashbaugh, M. S., Benguria, R. D.: Log-concavity of the ground state of Schrödinger operators: A new proof of the Baumgartner-Grosse-Martin inequality. Phys. Lett. 131A, 273-276(1988)

3. Ashbaugh, M. S., Benguria, R. D.: Proof of the Payne-Pólya-Weinberger conjecture. Bull. Am. Math. Soc. 25, 19-29 (1991)

4. Ashbaugh, M. S., Benguria, R. D.: Sharp bound for the ratio of the first two eigenvalues of Dirichlet Laplacians and extensions. Ann. Math (to appear)

5. Elbert, Á., Laforgia, A.: Monotonicity properties of the zeros of Bessel functions. SIAM J. Math. Anal. 17, 1483-1488 (1986)

6. Gori, L. N.-A., Laforgia, A., Muldoon, M. E.: Higher monotonicity properties and inequalities for zeroes of Bessel functions. Proc. Am. Math. Soc. 112, 513-520 (1991)

7. Ismail, M. E. H., Muldoon, M. E.: On the variation with respect to a parameter of zeros of Bessel and $q$-Bessel functions. J. Math. Anal. Appl. 135, 187-207 (1988)

8. Korevaar, N. J.: Capillary surface convexity above convex domains. Indiana Univ. Math. J. 32, 73-81 (1983)

9. Korevaar, N. J.: Convex solutions to nonlinear elliptic and parabolic boundary value problems. Indiana Univ. Math. J. 32, 603-614 (1983)

10. Lorch, L.: Elementary comparison techniques for certain classes of Sturm-Liouville equations, pp. 125-133. In: Differential Equations, Proceedings from the Uppsala 1977 International Conference on Differential Equations, Held at Uppsala, Sweden April 18-23, 1977, Berg, G., Essén, M., Pleijel, A. (eds.), Stockholm: Almqvist and Wiksell International 1977

11. Lorch. L.: Turánians and Wronskians for the zeros of Bessel functions, SIAM J. Math. Anal. 11, 223-227 (1980)

12. Payne, L. E., Pólya, G., Weinberger, H. F.: Sur le quotient de deux fréquences propres consécutives. Comptes Rendus Acad. Sci. Paris 241, 917-919 (1955)

13. Payne, L. E., Pólya, G., Weinberger, H. F.: On the ratio of consecutive eigenvalues. J. Math. Phys. 35, 289-298 (1956)

14. Watson, G. N.: A Treatise on the Theory of Bessel Functions, sec. ed. Cambridge: Cambridge University Press 1944

Communicated by B. Simon 